\title{
Slipped capital femoral epiphysis: an epidemiological Nationwide study in Italy from 2001 to 2015
}

Umile Giuseppe Longo ${ }^{1 *}$ D, Rocco Papalia ${ }^{1}$, Sergio De Salvatore ${ }^{1}$, Laura Ruzzini², Vincenzo Candela ${ }^{1}$, \|laria Piergentili ${ }^{1}$, Leonardo Oggiano ${ }^{2}$, Pier Francesco Costici ${ }^{2}$ and Vincenzo Denaro ${ }^{1}$

\begin{abstract}
Background: Slipped capital femoral epiphysis (epiphysiolysis of the femoral head, SCFE) is the most common pediatric hip disease in 10-14 years old children. The most used procedure to correct a stable form of SCFE is in situ pinning. Instead, the proper treatment for unstable forms is controversial. The first purpose of this study was to estimate annual admissions for SCFE in Italian patients from 2001 to 2015, basing on the hospitalization reports. The second aim was to assess the difference between regions regarding SCFE procedures. Lastly, a statistical prediction of the volume of SCFE procedures performed in Italy based on data from 2001 to 2015 was performed.

Methods: Data of this study were collected from the National Hospital Discharge Reports (SDO) reported at the Italian Ministry of Health regarding the years of this paper. The yearly number of hospital admission for SCFE, the percentage of males and females, the average age, days of hospitalization, primary diagnoses and primary procedures in the whole Italian population were calculated using descriptive statistical analyses.

Results: From 2001 to 2015, 4893 hospitalizations for SCFE were recorded in Italy, with a mean incidence of 2.9 (cases/100.000 inhabitants). The majority of patients treated by SCFE were males (70.6\%).

Conclusion: National health statistics for SCFE are attractive for an international audience, as different approaches to screening are reported between countries. These differences allow comparing outcomes internationally. Moreover, sharing national statistics and correlating those to other countries protocols, could be helpful to compare outcomes for different procedures internationally. However, further studies are required to understand the specific reasons for regional variation for SCFE procedures in Italy.
\end{abstract}

Level of evidence: III

Keywords: Epiphysiolysis, Slipped capital femoral epiphysis, SCFE, In situ fixation, Dunn procedure, Triplane proximal osteotomy, Epidemiology, Surgery, Young

\footnotetext{
* Correspondence: g.longo@unicampus.it

'Department of Orthopedic and Trauma Surgery, Campus Bio-Medico University of Rome, Rome, Italy

Full list of author information is available at the end of the article
}

(c) The Author(s). 2021 Open Access This article is licensed under a Creative Commons Attribution 4.0 International License, which permits use, sharing, adaptation, distribution and reproduction in any medium or format, as long as you give appropriate credit to the original author(s) and the source, provide a link to the Creative Commons licence, and indicate if changes were made. The images or other third party material in this article are included in the article's Creative Commons licence, unless indicated otherwise in a credit line to the material. If material is not included in the article's Creative Commons licence and your intended use is not permitted by statutory regulation or exceeds the permitted use, you will need to obtain permission directly from the copyright holder. To view a copy of this licence, visit http://creativecommons.org/licenses/by/4.0/ The Creative Commons Public Domain Dedication waiver (http://creativecommons.org/publicdomain/zero/1.0/) applies to the data made available in this article, unless otherwise stated in a credit line to the data. 


\section{Background}

Slipped Capital Femoral Epiphysis (also known as Epiphysiolysis of the femoral head, SCFE) is the most common pediatric hip disease that affects patients 10-14 years old [1,2]. SCFE is defined by posterior and inferior displacement (through the epiphyseal plate) of the proximal femoral epiphysis with the metaphysis. Each year in the USA, approximately 10.8 cases per 100.000 children of SCFE occurs [3, 4], and $18-50 \%$ are bilateral [5]. A timely diagnosis is challenging due to the relative frequency of the disease and the lack of significant symptoms [6]. Pain along with the adductor muscle (groin pull is uncommon in adolescents) is the most frequent symptom. The aetiology is multifactorial (endocrine diseases, hypogonadism, panhypopituitarism, growth spurts), but pediatric obesity represents the most relevant risk factor [7, 8]. SCFE is classified (using radiography and clinics) as stable or unstable forms based on the stability of the femoral physis and the capability to weight-bearing [9]. The former is treated using in situ closed screw fixation; instead, open reduction and fixation are usually adopted for unstable forms [10]. However, the proper treatment for unstable forms is still debated [11-13]. The major problem of this disease is the rapidity of diagnosis and the timing of surgery. The decision between conservative or operative treatment could also be influenced by geographical factors [14]. The prevalence of SCFE surgery in Europe is not fully defined, and only Sweden reported the nationwide incidence of this disease [15]. National health statistics for SCFE are attractive for an international audience, as different approaches to screening are reported between countries (type of screening, method of classification, mean age at the time of screening, diagnosis and subsequent treatment protocols). These differences allow comparing outcomes internationally. Moreover, sharing national statistics and correlating those to other countries protocols, could be helpful to compare outcomes for different procedures internationally.

This study was conducted from 2001 to 2015, based on official data source as hospitalization records. The principal purpose is to evaluate the yearly number of SCFE surgeries in Italy. The second purpose is to assess geographical variation in hospitalization for SCFE between three macro-areas of Italy (North, Center and South). Finally, a statistical projection of the volume of SCFE procedures in the next 5 years was performed.

\section{Methods}

Data of this study were collected from the National Hospital Discharge Reports (SDO) reported at the Italian Ministry of Health regarding the years of this paper (2001-2015). These reports provided data concerning all hospital admission occurring in Italy, both from public and private institutions. In Italy, the regional authorities are responsible for organizing and supervising healthcare services delivered through local structures (public or private). Data on the healthcare services are collected by hospitals and periodically sent to the Ministry of Health [16]. These data are anonymous and described the patient's age, sex, residence, the region of hospital admission, days of stays, diagnoses and procedures [17]. Population data were obtained from the National Institute for Statistics (ISTAT) for each year [18]. Epiphysiolysis was defined by the following International Classification of Diseases, Ninth Revision, Clinical Modification (ICD-9-CM) with the diagnosis code: 732.2. Since SCFE procedures for patients over 19 years were only 263 over the 15 -year study period, the study was restricted to the patients with $0-19$ years of age to avoid underestimation.

\section{Macro-areas of Italy}

Italy is divided into three macro-areas: North, Center and South-and-islands. The former includes four regions in the Western part (Aosta Valley, Liguria, Lombardy and Piedmont) and five in the Eastern part (Autonomous Province of Trento, Autonomous Province of Bolzano, Friuli-Venezia Giulia, Emilia-Romagna and Veneto). The Center counts four countries (Lazio, Marche, Tuscany and Umbria). The latter part of Italy includes five regions (Apulia, Basilicata, Calabria,

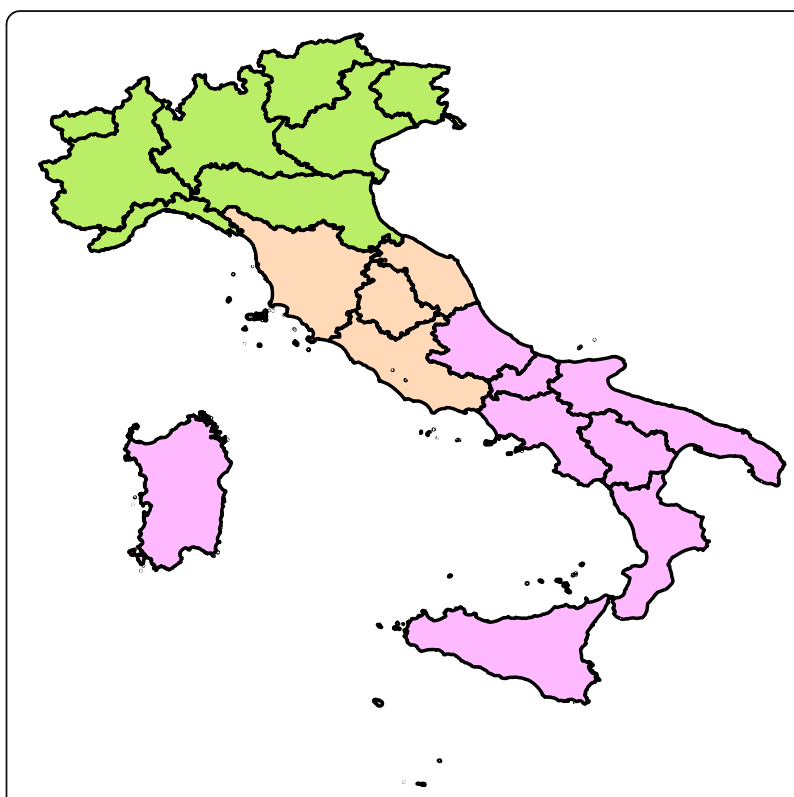

Fig. 1 Macro-areas of Italy are the North, the Centre and the South. (The figure was made using $\mathrm{R}$ software version i368 4.0.3) 
Number of SCFE procedures from 2001 to 2015

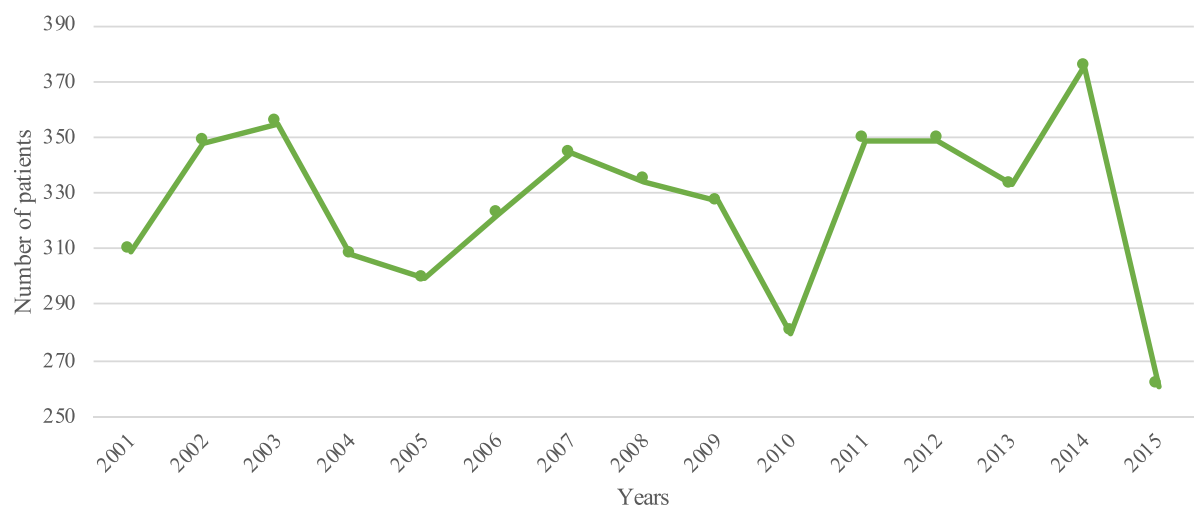

Fig. 2 Number of SCFE procedures from 2001 to 2015

Campania and Molise) and the islands (Sardinia and Sicily) (Fig. 1).

\section{Population}

The authors divided the patients for macro-area of domicile (North, Centre and South). The patients were divided into two groups (named "regional" and "extra-regional surgeries") to identify the presence of possible migratory flux. The former includes patients that were treated in the same macro-area of their domicile. Instead, the extra-regional group included patients who migrated from their domicile and were treated in other macro-areas.

\section{Statistics}

The authors used a descriptive statistical analysis were estimate the yearly number of SCFE procedures, the percentage of males and females, the average age, days of hospitalization, primary diagnoses and primary procedures in Italy. The annual adult population size obtained from ISTAT, a statutory electronic national population register, was used to calculate the incidence rates [19]. The incidence was based on the size of the whole Italian population of patients under 19 years old. To find statistical differences between years or sex, the linear regression analysis and the Mann-Whitney U Test were used, as applicable. The Exponential Smoothing (ETS) algorithm without seasonality was used to assess the forecast model. The Statistical Package for Social Sciences (SPSS) version 26 was used for this data analysis. Tables, graphs and forecast were performed using Excel (Microsoft) software.

\section{Results}

Population

During the study period, 4893 SCFE procedures were performed in Italy (Fig. 2), representing an incidence of
2.9 procedures for every 100,000 Italian inhabitants $(0-$ 19 years old). From 2001 to 2015, the incidence of operations slight decreased from 2.8 to 2.3 per 100,000 person-years $0-19$ years old without statistically significant result $(p=0.5)$ (Table 1$)$.

Over the study period, the 10-14-year age group reported the highest incidence of surgeries (Fig. 3).

Males represented the majority of patients, both in total and over the years (females $29.4 \%$ and males $70.6 \%$ ) (Fig. 4). No statistically significant differences in sex trend during the years were found $(p=0.4)$.

From 2001 to 2015, the average age of patients was $12.55 \pm 2.2$. During the entire period, the average age of males was always higher than in females (mean age of

Table 1 Incidence of SCFE procedures per 100'000 resident from 2001 to 2015

\begin{tabular}{llll}
\hline Years & Frequency & Percent & Incidence \\
\hline $\mathbf{2 0 0 1}$ & 309 & 6.3 & 2.8 \\
$\mathbf{2 0 0 2}$ & 348 & 7.1 & 3.2 \\
$\mathbf{2 0 0 3}$ & 355 & 7.3 & 3.2 \\
$\mathbf{2 0 0 4}$ & 308 & 6.3 & 2.8 \\
$\mathbf{2 0 0 5}$ & 299 & 6.1 & 2.7 \\
$\mathbf{2 0 0 6}$ & 322 & 6.6 & 2.9 \\
$\mathbf{2 0 0 7}$ & 344 & 7.0 & 3.0 \\
$\mathbf{2 0 0 8}$ & 334 & 6.8 & 2.9 \\
$\mathbf{2 0 0 9}$ & 327 & 6.7 & 2.9 \\
$\mathbf{2 0 1 0}$ & 280 & 5.7 & 2.4 \\
$\mathbf{2 0 1 1}$ & 349 & 7.1 & 3.1 \\
$\mathbf{2 0 1 2}$ & 349 & 7.1 & 3.1 \\
$\mathbf{2 0 1 3}$ & 333 & 6.8 & 2.9 \\
$\mathbf{2 0 1 4}$ & 375 & 7.7 & 3.3 \\
$\mathbf{2 0 1 5}$ & 261 & 5.3 & 2.3 \\
Total & 4893 & 100.0 & 2.9 \\
\hline
\end{tabular}




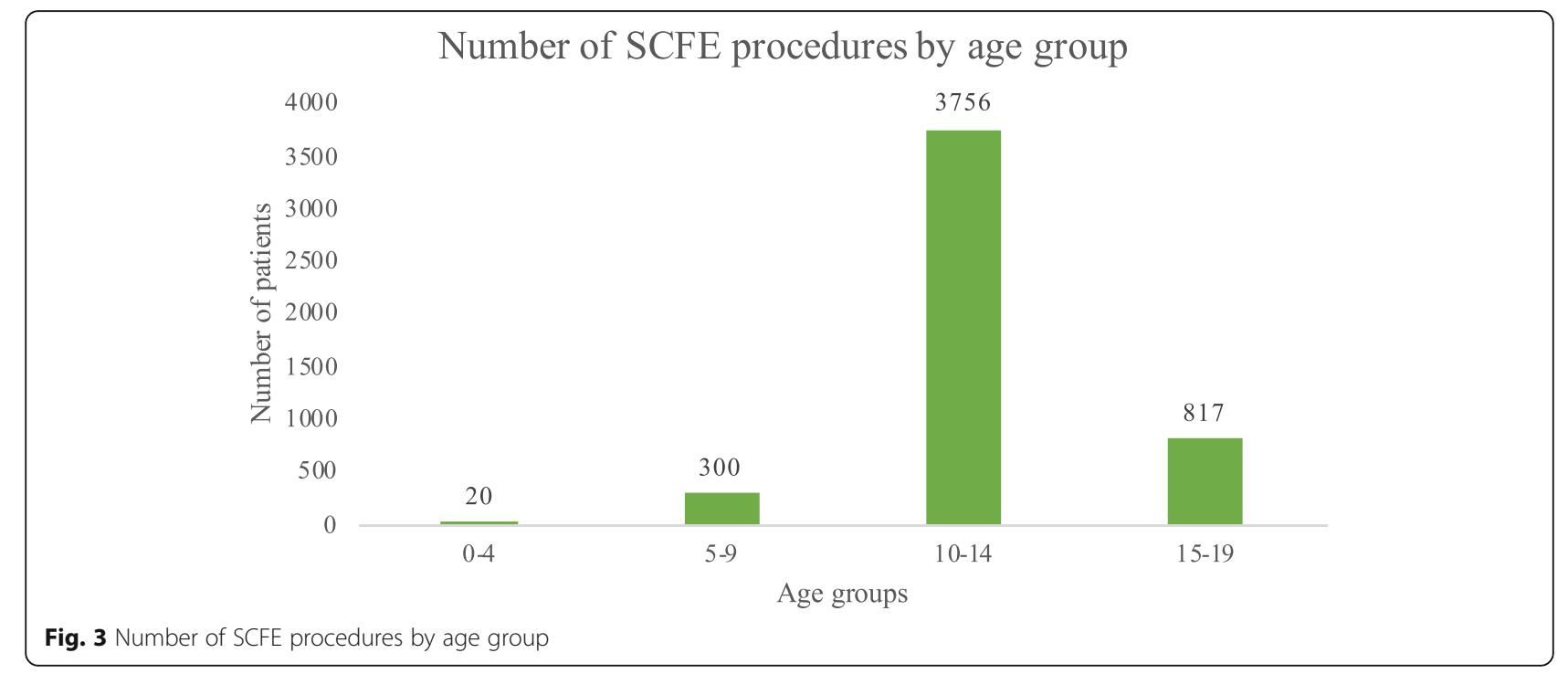

females $11.5 \pm 2$ and mean age of males $13 \pm 2 ; p<0.001$ ) (Fig. 5).

\section{Days of hospitalizations}

The mean length of days of hospitalization was 4.97 days (range 1-62 days). Males had more days of admission than females (M: 4.97 and F: 4.95 days; $p=0.8$ ). A general trend of decrease in days of hospitalization in both groups was observed ( $p=0.001$ ) (Fig. 6).

Patients aged from 5 to 9 had more days of hospitalization on average. Differentiating by sex, males with a higher number of days of hospitalization are between 10 and 14 years old, while women between 5 and 9 years old (Fig. 7).

\section{Region of admission and migratory flow}

Regarding the regional distribution, 2422 cases of SCFE procedures were performed in the North (49.5\%), 868 (17.7\%) in the Center, and 1603 (32.8\%) in the South. Of 4893 SCFE procedures performed in Italy during the study period, data on the patient's domicile was not available for 20 patients; therefore, only 4873 procedures were included in the analysis. From 2001 to 2015, 1491 patients (30.6\%) lived in the North, 823 patients (16.9\%) in the Center, and 2559 patients (52.5\%) in the South. Regional surgeries were $99.1 \%$ in the North, $77.9 \%$ in the Center and $61.9 \%$ in the South. The highest rate of extra-regional surgeries was recorded for patients that moved from the South to the North (29.7\%) and from

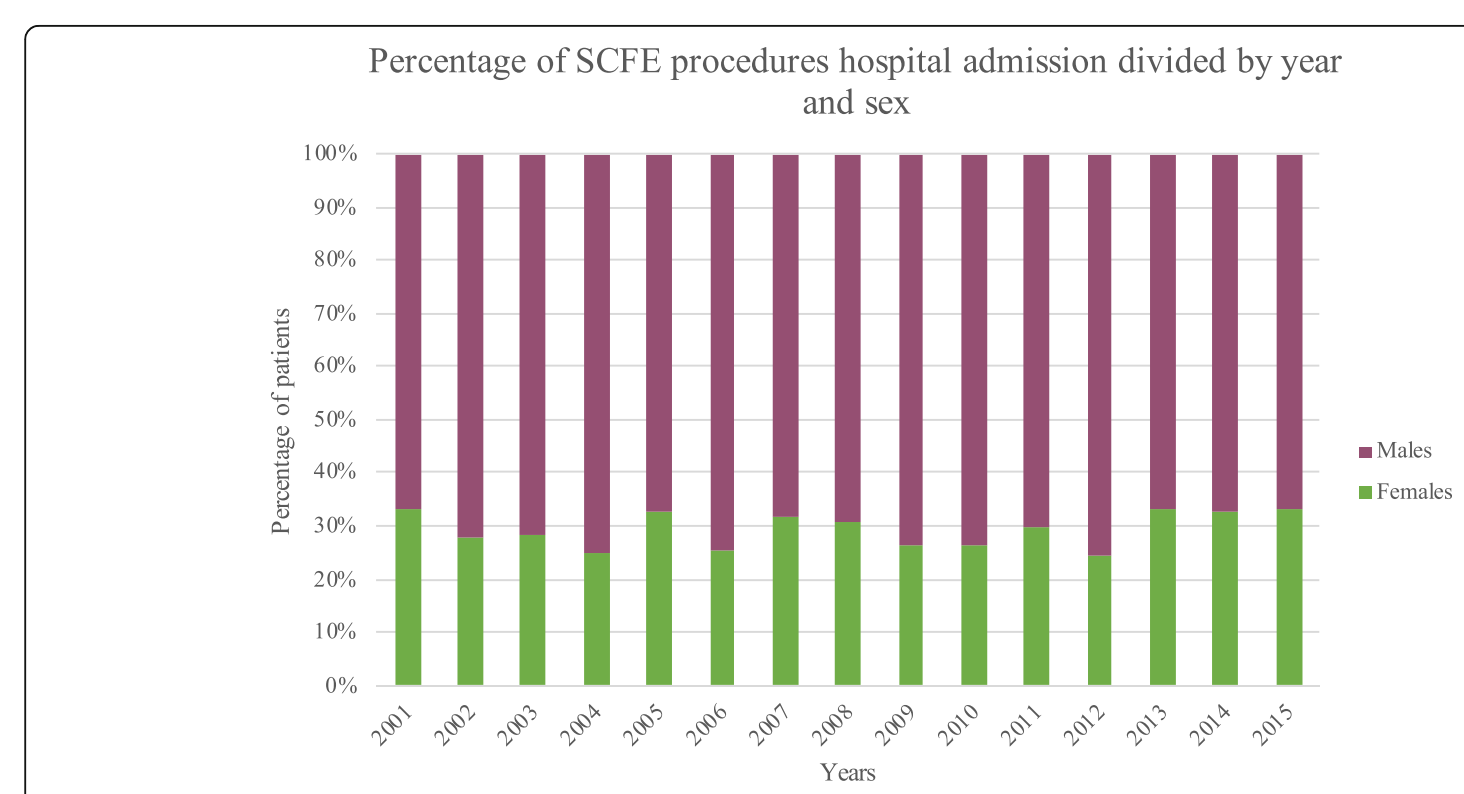

Fig. 4 Percentage of SCFE procedures hospital admission divided by year and sex 


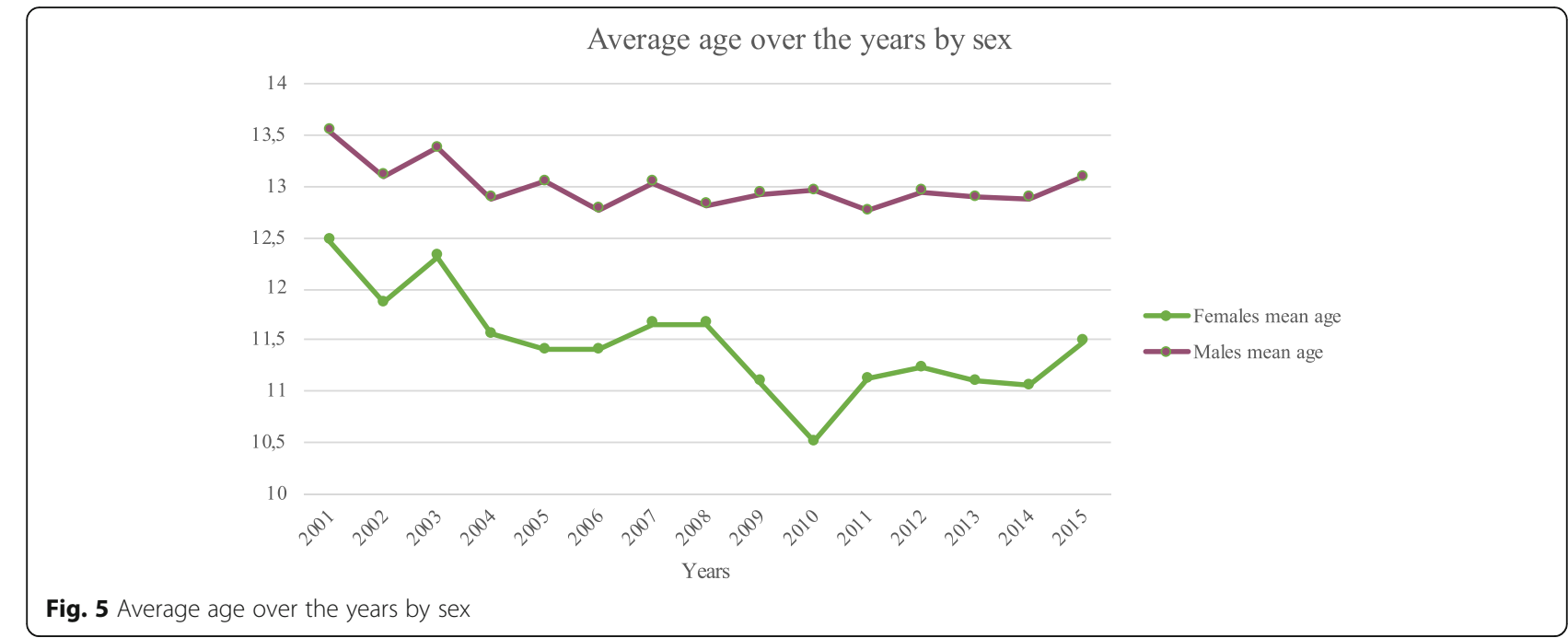

the Center to the North (21.0\%). All the data are reported in Table 2.

\section{Procedure performed and admission diagnosis codes}

During the 15-year study period, the main primary diagnoses were "Nontraumatic slipped upper femoral epiphysis" (84.1\%), "Aftercare involving internal fixation device" (3.8\%), "Other orthopaedic aftercare" (3.6\%) and "Encounter for removal of internal fixation device" $(2.4 \%)$. The main primary procedures performed are reported in Fig. 8.

\section{Projection model}

The projection model indicates a stable volume of hospitalization for SCFE procedures (Fig. 9). The projection model showed that the demand for SCFE procedures hospital admissions was estimated to remain unchanged from 261 in 2015 to 278 by 2025 .

\section{Discussion}

Epiphysiolysis of the femoral head is the most common hip disease in the pediatric population $[13,20]$, with an incidence of $0.2-0.3$ per 100,000 children aged 10-14 years [1]. The aetiology of SCFE is multifactorial and includes endocrine disorders, growth spurs and obesity [2, $4,7,21-23]$. History of trauma to the hip is uncommon [9]. The most common symptoms are pain and limping localized to the hip, groin, thigh or knee [24, 25]. A precise and rapid diagnosis is challenging due to the differential causes of hip pain in young patients [11]. Apophyseal avulsion fracture or apophysitis of the anterosuperior and anteroinferior iliac spine; septic arthritis and adductor muscle strain need to be excluded in these patients [4, 26]. Moreover, also transient synovitis, fractures and Legg-Calvè-Perthes should present similar symptoms. However, these conditions are uncommon in the SCFE age group $[4,26]$. A delayed diagnosis could avoid short and long-term complications as avascular

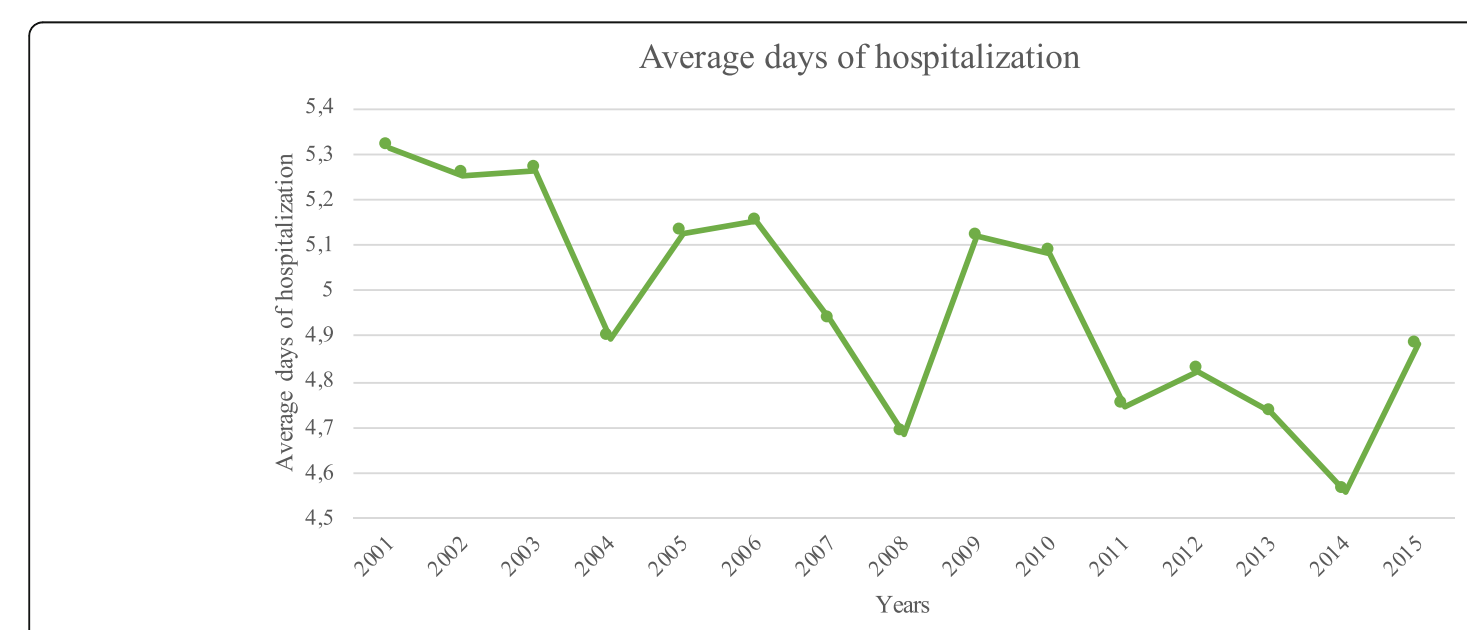

Fig. 6 Average days of hospitalization during the years 


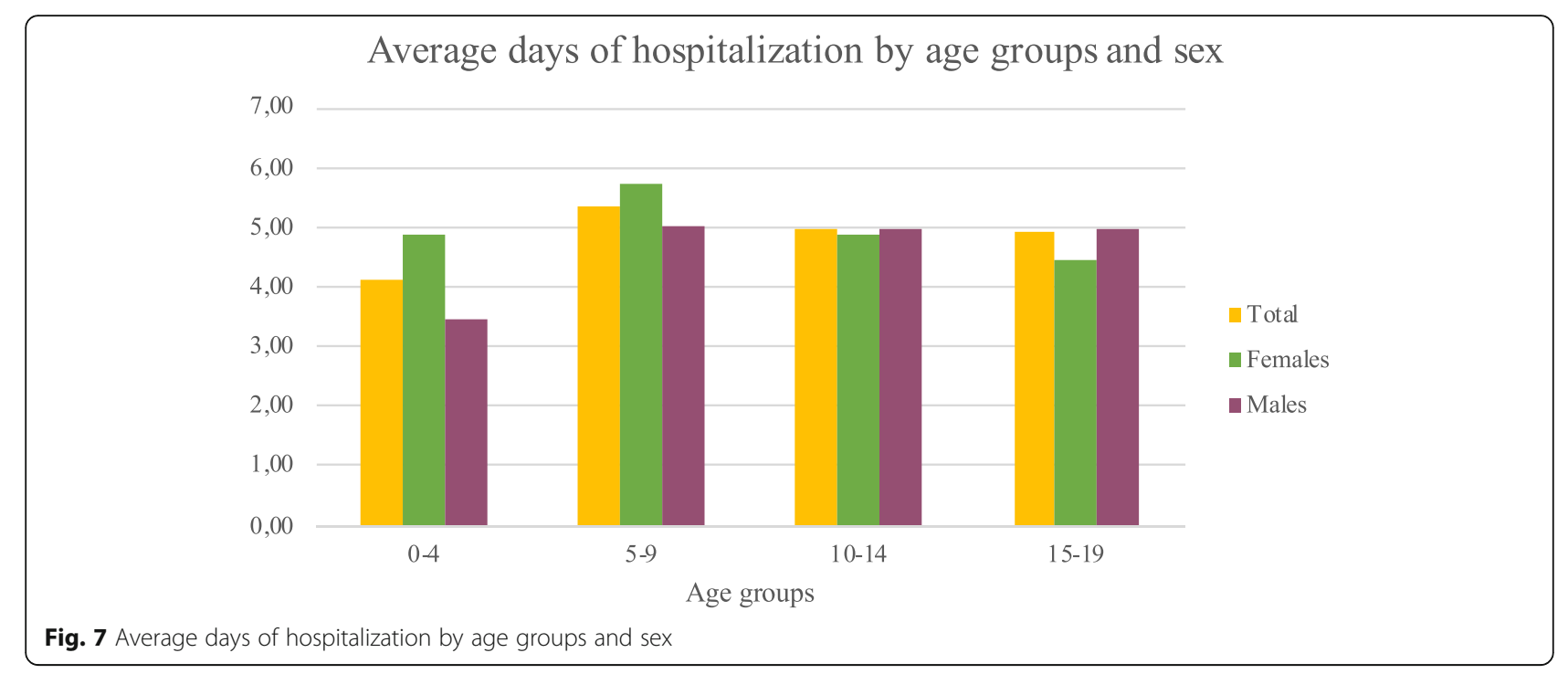

necrosis (AVN) of the femoral head and hip osteoarthritis, respectively [27]. Symptom's duration is used to classify SCFE in acute, acute on chronic and chronic forms. If symptoms present within 3 weeks, it is considered acute; instead, after 3 weeks, it is chronic [28]. The former is the most dangerous because related to a higher rate of AVN. Loder and colleagues [9] classified the stability of the physis based on the patient's capacity to bear weight, with or without crutches. Moreover, it is possible to evaluate SCFE by radiographical parameters using the Wilson and Southwick methods [9, 29, 30].
The initial step in the treatment of SCFE is to place the patient on non-weight bearing crutches or in a wheelchair [31]. It is mandatory to prevent slip progression and the insurgence of complications [32]. A closed reduction should not be attempted because it can result in AVN caused by the restricted blood supply to the epiphysis [33, 34]. Some authors recommend the prophylactic treatment of the contralateral hip, but there is no consensus concerning this topic [35]. There is a lack of high-quality literature on SCFE surgical management. However, based on the current literature, the best

Table 2 Analysis of migratory flows by macro-region 2001-2015

\begin{tabular}{|c|c|c|c|}
\hline \multicolumn{4}{|c|}{ Analysis of migratory flows by macro-region 2001-2015 } \\
\hline Macroregion of residence & Macroregion of hospitalization & Frequency & Percent \\
\hline \multirow[t]{4}{*}{ Unspecified } & Center & 5 & 25.0 \\
\hline & North & 10 & 50.0 \\
\hline & South & 5 & 25.0 \\
\hline & Total & 20 & 100.0 \\
\hline \multirow[t]{4}{*}{ Center } & Center & 641 & 77.9 \\
\hline & North & 173 & 21.0 \\
\hline & South & 9 & 1.1 \\
\hline & Total & 823 & 100.0 \\
\hline \multirow[t]{4}{*}{ North } & Center & 8 & 0.5 \\
\hline & North & 1478 & 99.1 \\
\hline & South & 5 & 0.3 \\
\hline & Total & 1491 & 100.0 \\
\hline \multirow[t]{4}{*}{ South } & Center & 214 & 8.4 \\
\hline & North & 761 & 29.7 \\
\hline & South & 1584 & 61.9 \\
\hline & Total & 2559 & 100.0 \\
\hline
\end{tabular}




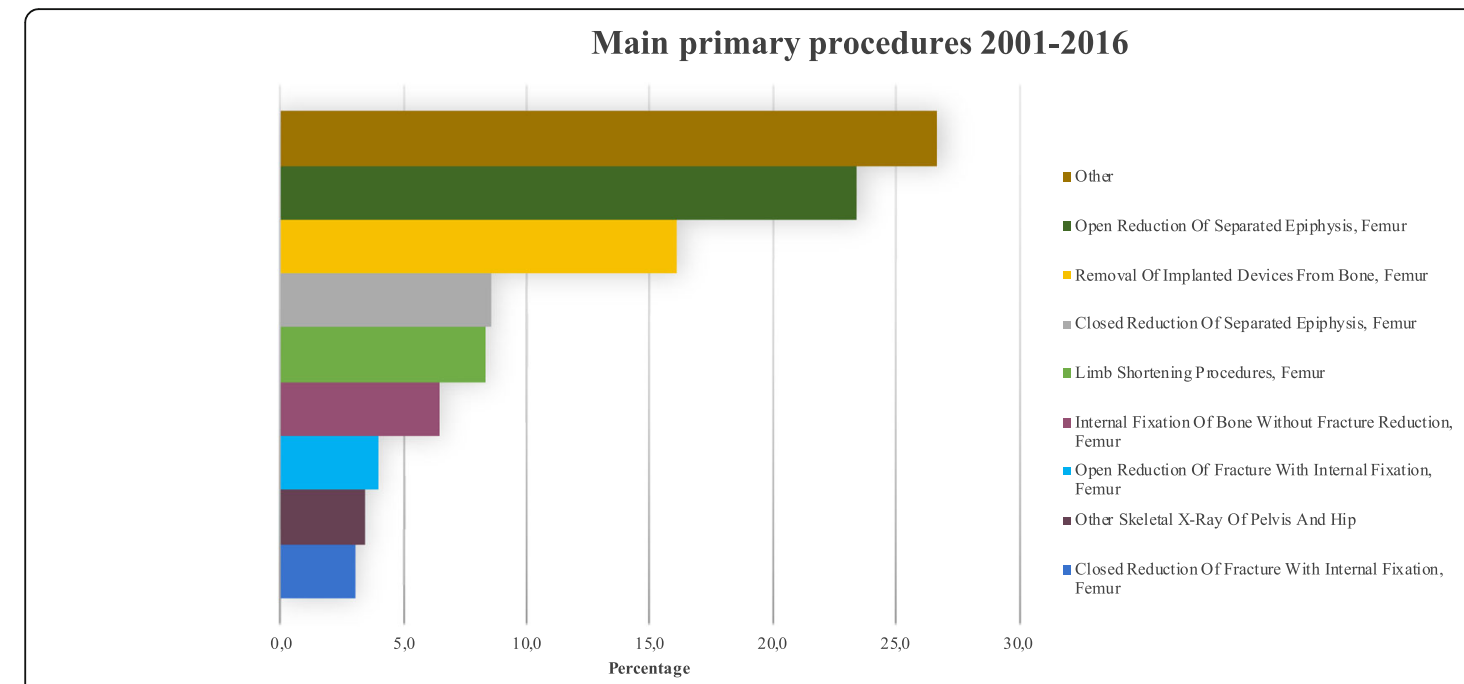

Fig. 8 Main primary procedures for SCFE from 2001 to 2015

treatment for stable SCFE is in situ pinning with a single screw, performed regardless of the timing of presentation [36]. The unstable SCFE is related to a higher risk of osteonecrosis (20-50\% of cases) [37-39], but the proper treatment and the timing associated with the lowest risk of AVN are still debated [11,36].. The technique described by Parsch and colleagues (open capsulotomy and partial reduction) seems to be the most promising, reporting a low rate of AVN [40]. Moreover, the modified Dunn procedure historically reported satisfactory outcomes with a low rate of necrosis, but it is widely influenced by the surgeon's technique and skills [36].

The most relevant complications of SCFE are AVN; degenerative osteoarthritis; acute loss of cartilages known as chondrolysis (reported after SCFE surgery or in untreated SCFE); femoroacetabular impingement [3, $12,41-43]$. The rate of AVN varies in the literature, but it is usually more frequent in unstable SCFE compared to stable forms [36]. Loder and colleagues [9] reported an AVN incidence of 0 and $47 \%$ for stable and unstable SCFE forms, respectively. However, recent literature reported an incidence of AVN between 0 and 3.3\% for stable forms and $23.9 \%$ for unstable forms [32].

SCFE is a relevant disease in the pediatric population and deserves to be known by clinicians. The main diagnosis code used for this analysis was 732.2 (Epiphysiolysis). The main procedures performed for SCFE were the "Open Reduction Of Separated Epiphysis, Femur" (23.4\%), followed by "Limb shortening Procedures, Femur" (8.3\%). In Italy, from 2001 to 2015, the mean incidence of hospital admission for SCFE was 2.9 for every 100,000 Italian inhabitants $0-19$ years old. The majority of patients were males of the 10-14 years age group, in line with the Swedish results, as reported by Herngren et al. [15]. Males reported a higher mean age compared to females $(p<0.001)$. The highest number of patients treated was domiciled in the South of Italy $(n=2559)$, followed by the North $(n=1491)$ and the Center $(n=$ 823). Otherwise, the highest number of procedures were

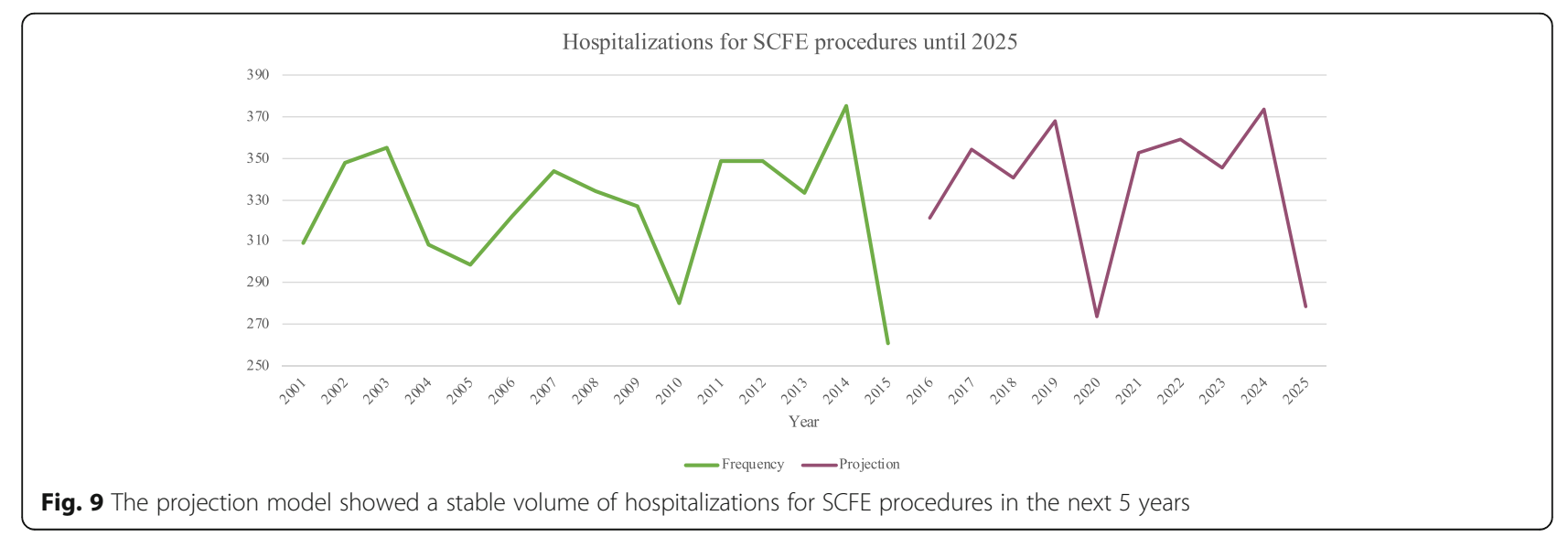


performed in the North $(n=2422)$ and the South $(n=$ 1603). The highest number of "extra-regional surgeries" were patients from the South that migrated to the North or the Center. Instead, patients from the North and the Center tended to be hospitalized in their macro-region of domicile. Moreover, patients from the South reported a higher rate of diagnosis of epiphysiolysis compared to the North and the South. A significant decrease in days of hospitalization during the study period was found, but further studies are required to identify possible explanations. The forecast model showed that the demand for SCFE hospital admissions was estimated to remain unchanged from 2015 to 2025.

To our knowledge, the only study on the SCFE surgery trend was performed by Herngren et al. [15]. However, only patients aged from 9 to 15 years were included, while a broader range of age was analysed in the present paper (0-19 years old). Moreover, the study period considered by Herngren et al. [15] was shorter compared to the present study (from 2007 to 2013 vs 2001 to 2015, respectively).

\section{Limitations}

This study is based on administrative data from different hospitals and macro-regions. The International Classification of Diseases 9 (ICD-9) was used for all the procedures reported. Otherwise, with the ICD-9, it was possible to use different codes for the same surgical procedure. This heterogeneity of codification could lead to an underestimation of our results. Secondly, the database has not been subject to internal validation. Moreover, we found 16.1\% of "Removal of Implanted Devices from Bone, Femur" within the procedures included. This data could lead to an overestimation of our results concerning the migratory flux and the total amount of procedures performed. Patients over 19 years old $(n=263)$ were excluded to avoid underestimation. Moreover, it was impossible to distinguish monoliteral vs bilateral screw fixation because ICD-9 did not fully code it. Lastly, this is a database study, and therefore it is not possible to define specific reasons for migratory flux. Further studies are required to define this trend precisely.

\section{Conclusions}

The incidence of surgery for SCFE in Italy is 2.9 cases/ 100,000 inhabitants of the same age group (from 2001 to 2015). The higher rate of hospitalization for SCFE was recorded in the South and the North. Epidemiological studies are helpful to understand the national variation of a specific surgical procedure and compare them with other countries. However, further studies are required to understand the specific reasons for regional variation for SCFE procedures in Italy.

\section{Acknowledgements}

We thank the Direzione Generale della Programmazione Sanitaria-Banca Dati SDO of the Italian Ministry of Health for the support in providing data for this research.

\section{Authors' contributions}

Conceptualization, U.G.L. and V.D.; Data curation, S.D.S., V.C. and L.R.; Formal analysis, S.D.S. and I.P..; Methodology, S.D.S., L.R.; Software, I.P. and S.D.S..; Supervision, L.O. and V.D.; Validation, P.F.C..; Visualization, L.O., R.P.., and V.D.; Writing-original draft, U.G.L., V.C. and S.D.S.:; Writing-review and editing, U.G.L., S.D.S., R.P.., and L.R. All authors have read and agreed to the published version of the manuscript.

\section{Funding}

This research received no external funding.

\section{Availability of data and materials}

The datasets used and/or analyzed during the current study are available from the corresponding author on reasonable request. The access to the database is on request. All data were obtained by the Direzione Generale della Programmazione Sanitaria-Banca Dati SDO of the Italian Ministry of Health.

\section{Declarations}

\section{Ethics approval and consent to participate}

The Institutional Review Board of Campus Bio-Medico University of Rome ruled that no formal ethics approval was required in this particular case. The access to the database is on request. All data were obtained by the Direzione Generale della Programmazione Sanitaria-Banca Dati SDO of the Italian Ministry of Health.

\section{Consent for publication \\ Not applicable.}

\section{Competing interests}

UGL is a member of the Editorial Board of BMC Musculoskeletal Disorders. The remaining authors declare that they have no conflict of interest.

\section{Author details}

${ }^{1}$ Department of Orthopedic and Trauma Surgery, Campus Bio-Medico University of Rome, Rome, Italy. ${ }^{2}$ Department of Surgery, Orthopedic Unit, Bambino Gesù Children's Hospital, Rome, Italy.

Received: 6 December 2020 Accepted: 6 June 2021

Published online: 22 June 2021

\section{References}

1. Otani T, Kawaguchi Y, Marumo K. Diagnosis and treatment of slipped capital femoral epiphysis: recent trends to note. J Orthop Sci Off J Jpn Orthop Assoc. 2018;23:220-8.

2. Gholve PA, Cameron DB, Millis MB. Slipped capital femoral epiphysis update. Curr Opin Pediatr. 2009;21(1):39-45. https://doi.org/10.1097/MOP.0b013e32 8320acea.

3. Lehmann CL, Arons RR, Loder RT, Vitale MG. The epidemiology of slipped capital femoral epiphysis: an update. J Pediatr Orthop. 2006;26(3):286-90. https://doi.org/10.1097/01.bpo.0000217718.10728.70.

4. Peck DM, Voss LM, Voss TT. Slipped capital femoral epiphysis: diagnosis and management. Am Fam Physician. 2017;95(12):779-84.

5. Riad J, Bajelidze G, Gabos PG. Bilateral slipped capital femoral epiphysis: predictive factors for contralateral slip. J Pediatr Orthop. 2007;27(4):411-4. https://doi.org/10.1097/01.bpb.0000271325.33739.86.

6. Krauspe R. Epiphyseolysis capitis femoris. Orthopade. 2019;48:643.

7. Reichelt A, Rütt A. Etiology of epiphysiolysis (epiphysiolisthesis) of the femur head. Arch Orthop Unfallchir. 1969;67(1):28-38. https://doi.org/10.1007/ BF00417137.

8. Villani C, Mantegna N, Chiozzi F, De Stefanis P, Persiani P. Epiphysiolysis of the hip: relationship between etiopathogenesis and hormone status. Chir Organi Mov. 2000;85:409-12.

9. Loder RT, Richards BS, Shapiro PS, Reznick LR, Aronson DD. Acute slipped capital femoral epiphysis: the importance of physeal stability. J Bone Joint 
Surg Am. 1993;75(8):1134-40. https://doi.org/10.2106/00004623-19930800000002.

10. Aprato A, Conti A, Bertolo F, Massè A. Slipped capital femoral epiphysis: current management strategies. Orthop Res Rev. 2019;11:47-54. https://doi. org/10.2147/ORR.S166735.

11. Sucato DJ. Approach to the hip for SCFE: the north American perspective. J Pediatr Orthop. 2018;38(Suppl 1):S5-12. https://doi.org/10.1097/BPO. 0000000000001183

12. Lykissas MG, McCarthy JJ. Should all unstable slipped capital femoral epiphysis be treated open? J Pediatr Orthop. 2013;33(Suppl 1):S92-8 https://doi.org/10.1097/BPO.0b013e318274f716.

13. Mathew SE, Larson AN. Natural History of Slipped Capital Femoral Epiphysis. J Pediatr Orthop. 2019;39(Issue 6, Supplement 1 Suppl 1):S23-7.

14. Loder RT, Schneble CA. Seasonal variation in slipped capital femoral epiphysis: new findings using a National Children's hospital database. J Pediatr Orthop. 2019;39(1):e44-9. https://doi.org/10.1097/BPO. 0000000000001074

15. Herngren B, Stenmarker M, Vavruch L, Hagglund G. Slipped capital femoral epiphysis: a population-based study. BMC Musculoskelet Disord. 2017;18(1): 304. https://doi.org/10.1186/s12891-017-1665-3.

16. Skrami E, Carle F, Villani S, Borrelli P, Zambon A, Corrao G, et al. Availability of real-world data in Italy: a tool to navigate regional healthcare utilization databases. Int J Environ Res Public Health. 2019;17(1). https://doi.org/10.33 90/ijerph17010008.

17. Longo UG, Salvatore G, Locher J, Ruzzini L, Candela V, Berton A, et al. Epidemiology of Paediatric shoulder dislocation: a Nationwide study in Italy from 2001 to 2014. Int J Environ Res Public Health. 2020;17(8). https://doi. org/10.3390/ijerph17082834.

18. Longo UG, Salvatore G, Rizzello G, Berton A, Ciuffreda M, Candela V, et al. The burden of rotator cuff surgery in Italy: a nationwide registry study. Arch Orthop Trauma Surg. 2017;137(2):217-24. https://doi.org/10.1007/s00402-01 6-2610-x.

19. Longo UG, Papalia R, De Salvatore S, Ruzzini L, Piergentili I, Oggiano L, et al. Trends in hospitalisation of Subtalar Joint Arthroereisis in Italy from 2009 to 2016. Foot Ankle Surg Off J Eur Soc Foot Ankle Surg 2021.

20. Dobbe AM, Gibbons PJ. Common paediatric conditions of the lower limb. J Paediatr Child Health. 2017;53(11):1077-85. https://doi.org/10.1111/jpc.13 756.

21. Georgiadis AG, Zaltz I. Slipped capital femoral epiphysis: how to evaluate with a review and update of treatment. Pediatr Clin N Am. 2014;61(6):111935. https://doi.org/10.1016/j.pcl.2014.08.001.

22. Hellmich HJ, Krieg AH. Slipped capital femoral epiphysis-etiology and pathogenesis. Orthopade. 2019;48(8):644-50. https://doi.org/10.1007/s00132019-03743-4.

23. Perry DC, Metcalfe D, Lane S, Turner S. Childhood obesity and slipped capital femoral epiphysis. Pediatrics. 2018;142(5):e20181067. https://doi.org/1 0.1542/peds.2018-1067.

24. Reynolds RA. Diagnosis and treatment of slipped capital femoral epiphysis. Curr Opin Pediatr. 1999;11(1):80-3. https://doi.org/10.1097/00008480-199902 000-00016.

25. Castillo C, Mendez M. Slipped capital femoral epiphysis: a review for pediatricians. Pediatr Ann. 2018;47(9):e377-80. https://doi.org/10.3928/193 82359-20180730-01.

26. Karkenny AJ, Tauberg BM, Otsuka NY. Pediatric hip disorders: slipped capital femoral epiphysis and Legg-Calvé-Perthes disease. Pediatr Rev. 2018;39(9): 454-63. https://doi.org/10.1542/pir.2017-0197.

27. Millis MB. SCFE diagnosis delayed among patients with referred pain. Pediatr. 2019;212:242. https://doi.org/10.1016/j.jpeds.2019.07.010

28. Bittersohl D, Bittersohl B, Westhoff B, Krauspe R. Slipped capital femoral epiphysis: clinical presentation, diagnostic procedure and classification. Orthopade. 2019;48(8):651-8. https://doi.org/10.1007/s00132-019-03767-w.

29. Jarrett DY, Matheney T, Kleinman PK. Imaging SCFE: diagnosis, treatment and complications. Pediatr Radiol. 2013;43(Suppl 1):S71-82.

30. Jones CE, Cooper AP, Doucette J, Buchan LL, Wilson DR, Mulpuri K, et al. Southwick angle measurements and SCFE slip severity classifications are affected by frog-lateral positioning. Skelet Radiol. 2018;47(1):79-84. https:// doi.org/10.1007/s00256-017-2761-z.

31. Rossiter DJ, Ahluwalia A, Vo P, Mapara R. The limping child: a systematic approach to assessment and management. Br J Hosp Med Lond Engl 2005. 2018:79:C150-3.
32. Naseem H, Chatterji S, Tsang K, Hakimi M, Chytas A, Alshryda S. Treatment of stable slipped capital femoral epiphysis: systematic review and exploratory patient level analysis. J Orthop Traumatol Off J Ital Soc Orthop Traumatol. 2017;18(4):379-94. https://doi.org/10.1007/s10195-017-0469-4.

33. Bartoszewski T, Mazurek T, Siwicka K, Samul G. Results of the treatment of the distal femoral epiphysiolysis. Chir Narzadow Ruchu Ortop Pol. 2007; 72(4):253-7.

34. Surgery for slipped capital femoral epiphysis in adolescents - PubMed. https://pubmed.ncbi.nlm.nih.gov/24397949/. Accessed 13 Aug 2020

35. Lim YJ, Lam KS, Lee EH. Review of the management outcome of slipped capital femoral epiphysis and the role of prophylactic contra-lateral pinning re-examined. Ann Acad Med Singap. 2008;37:184-7.

36. Daley E, Zaltz I. Strategies to avoid osteonecrosis in unstable slipped capital femoral epiphysis: a critical analysis review. JBJS Rev. 2019;7(4):e7. https:// doi.org/10.2106/JBJS.RWW.18.00129

37. McPartland TG, Sankar WN, Kim Y-J, Millis MB. Patients with unstable slipped capital femoral epiphysis have antecedent symptoms. Clin Orthop. 2013; 471(7):2132-6. https://doi.org/10.1007/s11999-013-3042-3.

38. Aronsson DD, Loder RT. Treatment of the unstable (acute) slipped capital femoral epiphysis. Clin Orthop. 1996:99-110.

39. Zaltz I, Baca G, Clohisy JC. Unstable SCFE: review of treatment modalities and prevalence of osteonecrosis. Clin Orthop. 2013;471(7):2192-8. https:// doi.org/10.1007/s11999-012-2765-x.

40. Parsch K, Weller S, Parsch D. Open reduction and smooth Kirschner wire fixation for unstable slipped capital femoral epiphysis. J Pediatr Orthop. 2009:29(1):1-8. https://doi.org/10.1097/BPO.0b013e31818f0ea3.

41. Boero S, Brunenghi GM, Carbone M, Stella G, Calevo MG. Pinning in slipped capital femoral epiphysis: long-term follow-up study. J Pediatr Orthop Part B. 2003;12:372-9.

42. Ziebarth K, Steppacher SD, Siebenrock KA. The modified Dunn procedure to treat severe slipped capital femoral epiphysis. Orthopade. 2019;48(8):668-76. https://doi.org/10.1007/s00132-019-03774-x.

43. Soni JF, Valenza WR, Uliana CS. Surgical treatment of femoroacetabular impingement after slipped capital femoral epiphysis. Curr Opin Pediatr. 2018;30(1):93-9. https://doi.org/10.1097/MOP.0000000000000565.

\section{Publisher's Note}

Springer Nature remains neutral with regard to jurisdictional claims in published maps and institutional affiliations.
Ready to submit your research? Choose BMC and benefit from:

- fast, convenient online submission

- thorough peer review by experienced researchers in your field

- rapid publication on acceptance

- support for research data, including large and complex data types

- gold Open Access which fosters wider collaboration and increased citations

- maximum visibility for your research: over $100 \mathrm{M}$ website views per year

At $\mathrm{BMC}$, research is always in progress.

Learn more biomedcentral.com/submissions 